\title{
New Reverberation Mapping Results from the Lick AGN Monitoring Project
}

\author{
Misty C. Bentz ${ }^{1}$ and the LAMP Collaboration \\ ${ }^{1} 4129$ Frederick Reines Hall, Department of Physics and Astronomy, \\ University of California, Irvine CA 92617, USA \\ Email: mbentz@uci.edu
}

\begin{abstract}
Eight new black hole masses have been derived from a recent reverberation-mapping experiment carried out at Lick Observatory. The masses lie in the range $\sim 10^{6}-10^{7} M_{\odot}$ and will allow us to extend the low end of AGN scaling relationships by a factor of $\sim 10$.
\end{abstract}

Keywords. galaxies: active, galaxies: nuclei, galaxies: Seyfert

We have recently completed a 64-night spectroscopic monitoring campaign at the Lick Observatory $3-\mathrm{m}$ Shane telescope with the aim of measuring the masses of the black holes in 12 nearby $(z<0.05)$ Seyfert 1 galaxies expected to have $10^{6} \lesssim M_{\mathrm{BH}} \lesssim 10^{7} M_{\odot}$. The well-studied AGN NGC 5548 was also included, for a total of 13 targets. Nine of the AGNs in the sample (including NGC 5548) showed optical variability of sufficient strength during the monitoring campaign (Walsh et al. 2009) to allow for a time lag to be measured between the continuum fluctuations and the delayed response in the broad emission lines, thus permitting virial black hole mass determinations from several optical emission lines in each object. The mass determined for NGC 5548 agrees well with previous determinations from 13 years of independent reverberation-mapping (RM) experiments, and the addition of eight new black hole mass measurements brings the RM sample to a total of 43 AGNs (Bentz et al. 2008, 2009).

Currently, the AGN $M_{\mathrm{BH}}-\sigma_{*}$ and $R_{\mathrm{BLR}}-L_{\mathrm{AGN}}$ relationships are not well constrained for $L_{5100}<10^{43} \mathrm{erg} \mathrm{s}^{-1}$ and $M_{\mathrm{BH}}<10^{7} M_{\odot}$. With the lower luminosities and masses of the LAMP AGNs (compared to previous RM targets), we will extend the range of the AGN $M_{\mathrm{BH}}-\sigma_{*}$ relationship using the LAMP reverberation masses and stellar velocity dispersions from spectra obtained at the Keck, Palomar, and Lick Observatories (Woo et al. 2010 , in preparation). We will also extend the range of the $R_{\mathrm{BLR}}-L_{\mathrm{AGN}}$ relationship by almost a decade after correcting the luminosities of the LAMP AGNs for the contribution from host-galaxy starlight with high-resolution Hubble Space Telescope images (Cycle 17 GO 11662, PI Bentz).

In addition, we find evidence for velocity-resolved time lag behavior across the broad Balmer line profiles in several of the AGNs. The variety of velocity-resolved behaviors seen in this sample argues for a diversity of broad line region parameters across the AGN population (Bentz et al. 2009, 2010).

\section{References}

Bentz, M. C., et al. 2008, ApJ, 689, L21

Bentz, M. C., et al. 2009, ApJ, 705, 199

Bentz, M. C., et al. 2010, in preparation

Walsh, J. L., et al. 2009, ApJS, 185, 156

Woo, J.-H. et al. 2010, in preparation 